\title{
Prediction And Verification of Wafer Surface Morphology in Ultrasonic Vibration Assisted Wire Saw (UAWS) Slicing Single Crystal Silicon Based On Mixed Material Removal Mode
}

\author{
Yan Wang \\ University of Shanghai for Science and Technology \\ Rui Wang ( $D$ 18301926280@163.com ) \\ University of Shanghai for Science and Technology \\ Shusheng Li \\ Taizhou Polytechnic College \\ Jianguo Liu \\ University of Shanghai for Science and Technology \\ Lixing Song \\ University of Shanghai for Science and Technology
}

\section{Research Article}

Keywords: Ultrasonic vibration, Surface morphology, Prediction, Mixed material removal mode

Posted Date: December 1st, 2021

DOI: https://doi.org/10.21203/rs.3.rs-949996/v1

License: (c) (i) This work is licensed under a Creative Commons Attribution 4.0 International License. Read Full License

Version of Record: A version of this preprint was published at The International Journal of Advanced Manufacturing Technology on April 18th, 2022. See the published version at https://doi.org/10.1007/s00170-022-09218-9. 


\title{
Prediction and Verification of Wafer Surface Morphology in \\ Ultrasonic Vibration Assisted Wire Saw (UAWS) Slicing Single
}

\section{Crystal Silicon Based on Mixed Material Removal Mode}

\author{
Yan Wang ${ }^{\text {a, }}$, Rui Wang ${ }^{\text {a }}$, Shusheng Li $^{\text {b }}$, Jianguo Liu ${ }^{\text {a }}$, Lixing Song ${ }^{\text {a }}$ \\ a School of Mechanical Engineering, University of Shanghai for Science and Technology, \\ Shanghai 200093, China \\ b Department of Mechanical and Electrical Engineering, Taizhou Polytechnic College, Taizhou \\ 225300, China \\ *Corresponding author, E-mail addresses: yanwang909909@163.com (Y. Wang)
}

\begin{abstract}
Monocrystalline silicon is one of the most important semiconductor materials, widely used in chip manufacturing, solar panels. Slicing is the first step in making chips and the surface quality of silicon wafers directly affects the quality of later processing and accounts for a large proportion in the chip manufacturing cost. Ultrasonic vibration assisted wire saw (UAWS) is an effective sawing process for cutting hard and brittle materials such as monocrystalline $\mathrm{Si}$, which can significantly improve the surface quality of silicon wafers. In order to further study the formation mechanism of the surface morphology of single crystal silicon sliced by UAWS, a new model for prediction of wafer surface morphology in UAWS slicing single crystal silicon based on mixed material removal mode is presented and verified in this paper. Firstly, the surface model of diamond wire saw tool is established by equal probability method. Then according to the equation of transverse vibration dynamics about the wire saw with ultrasonic excitation, the trajectory equation of arbitrary abrasive particles on the surface of wire saw is derived and analyzed. Thirdly, a new model for prediction of the wafer surface morphology based on mixed material removal mode is presented, which can be used to predict the wafer surface morphology of single crystal silicon sliced by UAWS. Finally, the prediction model is verified by UAWS slicing experiment, and the effects of slicing parameters such as wire saw speed, feed speed and workpiece rotate speed on the surface quality of silicon wafer were studied. It shows that the predicted wafer surface morphology and the experimental wafer surface morphology are similar in some characteristics, and the average error between the experimental and the theoretical values of the wafer surface roughness is $11.9 \%$, which verifies the validity of the prediction model.
\end{abstract}

Key words: Ultrasonic vibration; Surface morphology; Prediction; Mixed material removal mode

\section{Introduction}

Single crystal silicon is one of the most important semiconductor materials widely used in chipmanufacturing, solar panels and other equipment. Slicing is the first step in making chips and the surface quality of silicon wafers directly affects the quality of later processing and accounts for a large proportion in the chip manufacturing cost [1-3]. In slicing technology, wire saw slicing is a widely used technology, which first appeared in the 1990s. Ultrasonic vibration assisted wire saw (UAWS) slicing is a kind of composite processing technology which combines ultrasonic vibration 
and fixed abrasive diamond wire saw processing, and literatures have shown that ultrasonic vibration assisted wire saw slicing technology can obviously improve the surface quality of wafers, reduce the sawing force and raise the processing efficiency [4]. In order to further study the formation mechanism of the surface morphology of single crystal silicon sliced by UAWS, it is necessary to build a model to predict the surface morphology of the sliced wafers and analyze the effect of ultrasonic vibration and processing parameters on the surface morphology.

In order to investigate on the surface quality of workpiece sliced by wire saw, some theoretical and experimental studies have been studied by many scholars: Chung and Nhat [5] established a wire saw model, which generated the surface morphology of the workpiece according to the interaction between the abrasive particles and the workpiece. Experiments are carried out to verify the simulation results. Ge et al. [6] have conducted the experiment on KDP crystal sliced by resin bonded diamond wire saw. Experimental results show that the workpiece feed rate is the main affecting factor on surface quality of KDP crystals. Teomete [7] conducted an experimental parameter study to investigate the influence of process parameters on the wire bow angle, wire distributed load and surface roughness when wire saw cutting alumina ceramics. And process design recommendations for increasing efficiency of the wire saw cutting process while keeping the surface roughness constant are presented. The experiment of UAWS cutting monocrystalline SiC was conducted by Li et al. [8] on WXD170 reciprocating diamond wire saw cutting machine tool. An empirical model was developed for predicting the surface roughness when wire sawing $\mathrm{SiC}$ wafers and optimum process parameters for minimizing surface roughness are determined using the desirability functional approach. Gao et al. [9] conducted the slicing experiments of single crystal silicon. It indicates that the main material removal mode in the wire saw cutting single crystal silicon is brittle mode. Li et al. [10] found that when the position angle of abrasive particle is less than a critical angle $\theta \mathrm{c}$, the material removal mode of the abrasive particle is brittle removal and the brittle fracture will be generated on the wafer surface. Wang et al. [11] used the FEM/SPH coupling algorithm to simulate the material removal process of diamond abrasive particles cutting brittle optical materials, and revealed the material removal mechanism. Erick et al. [12] have investigated the influence of diamond wire sawing on surface integrity of monocrystalline silicon. It was found that with the increase in feed rate, the brittle mode is predominant and the silicon surface presents deeper and wider craters. With the increase of wire cutting speed, there were more regions formed in ductile mode. Yin et al. [13] established a mathematical model of diamond wire sawing based on the machining mechanism of brittle material removal and surface generation, and carried out a numerical calculation of the sawing process. It was found that it is more conducive to obtaining a crack-free sawn surface by reducing the workpiece feed speed or increasing the saw wire movement speed, and the workpiece feed speed has a greater impact on the sawn surface quality.

During the traditional wire saw slicing process, the abrasive particles fixed on the wire saw will continuously contact and rub with the workpiece, thus removing the workpiece material. In the ultrasonic vibration assisted wire saw slicing, the abrasive particles are contacted against the workpiece in an intermittent mode due to the application of ultrasonic excitation. Abrasive particles constantly hit the workpiece at a high frequency, resulting in brittle removal of materials. At the same time, the wafer surface morphology is formed by rubbing and grinding of some abrasive particles under ultrasonic excitation. In the formation of wafer morphology, most scholars only consider the ductile removal of wafers by abrasive particles. In fact, the manner of material removal of abrasive particles is different for those abrasive particles with different cutting depth. The 
brittleness removal mode of some abrasive particles will affect the final surface morphology of the wafer. Therefore, it is necessary to consider the influence of brittleness removal mode on wafer surface morphology when modeling.

In order to further study the formation mechanism of the surface morphology of single crystal silicon sliced by UAWS, a new model for prediction of wafer surface morphology in UAWS slicing single crystal silicon based on mixed material removal mode is presented and verified in this paper. Firstly, the surface model of diamond wire saw is established by equal probability method. Then according to the equation of transverse vibration dynamics about the wire saw with ultrasonic excitation, the trajectory equation of arbitrary abrasive particles on the surface of wire saw is derived. Thirdly, a new model for predicting the surface morphology of wafers based on the mixed material removal mode is proposed, which can be used to predict the surface morphology of single crystal silicon wafers cut by UAWS. Finally, a verification experiment is carried out. In addition, the prediction results and the experimental results are compared and analyzed. It shows that the predicted wafer surface morphology and the experimental wafer surface morphology are similar in some characteristics, and the average error between the experimental and predicted values of the wafer surface roughness value is $11.9 \%$, which verifies the validity of the prediction model.

\section{Surface model of wire saw tool}

\subsection{Principle of UAWS slicing}

The schematic of UAWS slicing is shown in Fig.1. The wire saw is wound between a wrapping droller, two tension wheels, two guide wheels, an ultrasonic guide wheel and an assistant guide wheel forming a closed loop. The machining coordinate system oxyz is established by taking the center of workpiece as the origin, the horizontal right direction as the positive direction of the $x$ axis and the vertical upward direction as the positive direction of the $y$ axis as shown in Fig.1. During the slicing process, the wire saw is stretched by the tension wheels with a pair of constant tension force $F_{T}$. The wire saw is moved at a linear speed $v_{s}$ reciprocally driven by the rotation of the wrapping droller. In the meantime, the wrapping droller, tension wheels, guide wheels, ultrasonic guide wheel and the assistant guide wheel are moved downward together along the negative direction of the $y$ axis at a same feed rate of $v_{w}$, while the workpiece is rotating about the $z$ axis which is the axis of workpiece itself at a rotate speed of $n_{w}$. The ultrasonic vibration is applied on the ultrasonic wheel along the $y$ axis with amplitude $A$ and frequency $f$. The workpiece is sliced by wire saw under ultrasonic excitation.

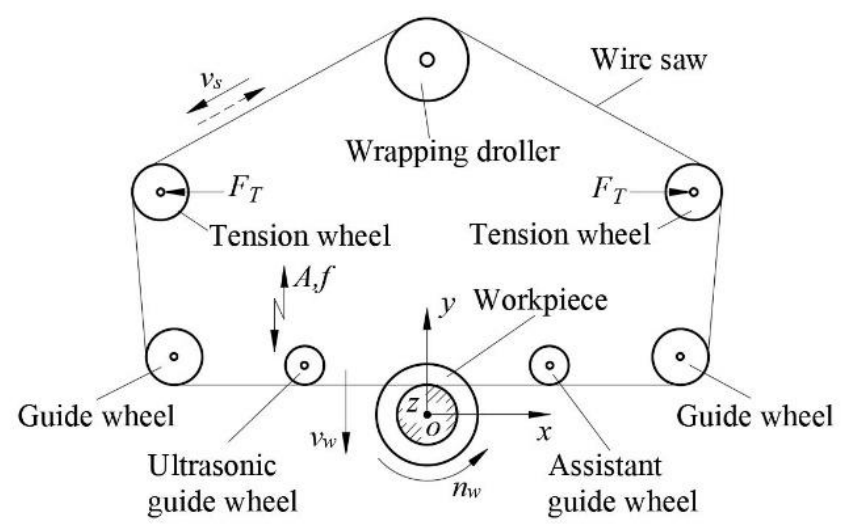

Fig.1 Schematic of UAWS slicing 
In order to simulate the UAWS slicing process and establish the prediction model of wafer surface morphology, the tool surface model should be established first.

\subsection{Establishment of surface model of wire saw tool}

The fixed diamond wire saw is an effective tool for cutting hard and brittle materials such as monocrystalline silicon and $\mathrm{SiC}$, which widely used in chip manufacturing industry [3]. The distances between abrasive particles are large, and bigger than the diameter of abrasive particles, So the general method of grinding wheel surface modeling cannot be adopted. Therefore, the equal probability method [4] is used to build the wire saw tool surface model.

Since the wire saw base is made of stainless-steel wire, it is assumed that the wire saw is an ideal cylinder. When modeling, the length of wire saw is $L$, the diameter of wire saw base is $D_{B}$, and the density of the wire saw is $\rho$. Then the number of abrasive particles on wire saw can be obtained, as shown in Eq. (1).

$$
N_{a b r}=\pi D_{B} L \rho
$$

For the convenience of calculation, the wire saw base surface is expanded into a plane for modeling. The plane is divided into several grids on average, and the number of nodes is:

$$
N=m \times n
$$

Where $m$ is the number of nodes in the axial direction of the wire saw and $n$ is the number of nodes in the circumferential direction of the wire saw. In order to ensure that the probability of abrasive particles on each node is equal, the relationship between $m$ and $n$ should be guaranteed as shown in Eq. (3).

$$
\frac{L}{m}=\frac{\pi D_{B}}{n}
$$

Diamond abrasive particles are inserted on the divided nodes randomly, and the probability of the presence of an abrasive particle at a node can be obtained from Eq. (4).

$$
P_{a b r}=\frac{\pi D_{B} L \rho}{N}=\frac{\pi D_{B} L \rho}{m \times n}
$$

In this paper, the geometrical shape of the abrasive particle is simplified into a cone shape [14], as shown in Fig.2. It is found that the size distribution of abrasive particles $d_{g}$ conforms to normal distribution [15] whose probability density function is given by:

$$
f\left(d_{g}\right)=\frac{1}{\sqrt{2 \pi \sigma}} \exp \left\{-\frac{\left[\mu+\log \left(d_{g}\right)\right]^{2}}{2 \sigma^{2}}\right\}
$$

with $\mu$ and $\sigma$ being the mean and standard deviation, respectively.

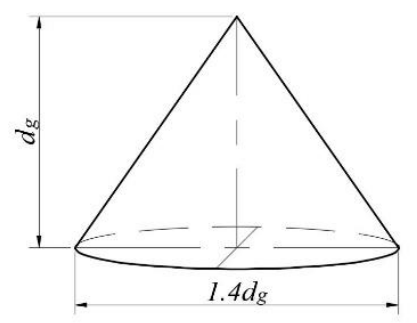

Fig.2 Simplified geometric model of the abrasive particle

A diamond wire saw was observed by the KEYENCE VXH6000 optical microscope to get some necessary parameters such as abrasive particle sizes. For a given abrasive particle size, the maximum size of the abrasive particle is $d_{\text {gmax }}$, and the minimum size of the abrasive particle is $d_{\text {gmin }}$. The mean size of the abrasive particle $d_{\text {gave }}$ is about $60 \mu \mathrm{m}$. At the same time, $d_{\text {gmax }}$ and $d_{\text {gmin }}$ are $70 \mu \mathrm{m}$ and $50 \mu \mathrm{m}$ respectively. The standard deviation $\sigma$ can be obtained by Eq. (6). 


$$
\sigma=\frac{d_{g \max }-d_{\text {gmin }}}{6}
$$

Therefore, the abrasive particle size distribution is assumed to follow normal distribution with the mean size of $60 \mu \mathrm{m}$ and the standard deviation $\sigma=3.33 \mu \mathrm{m}$. The research of Chung et al. [5] noted that it is difficult to control the size of such tiny diamond particles in practical process, so the influence of different abrasive particle size distribution on the prediction results in the modeling process is not considered. The destiny of abrasive particles is 60 grits $/ \mathrm{mm}^{2}$.

The thickness of the plating coating on the wire saw base is assumed to be $0.02 \mathrm{~mm}$. In order to simplify the model, it is considered that the expanded coating surface has the same area as the wire saw base surface. The wire saw tool topography can be generated as shown in Fig.3. According to the generated model, the position and the size of abrasive particles in the spreading plane of wire saw surface can be obtained.

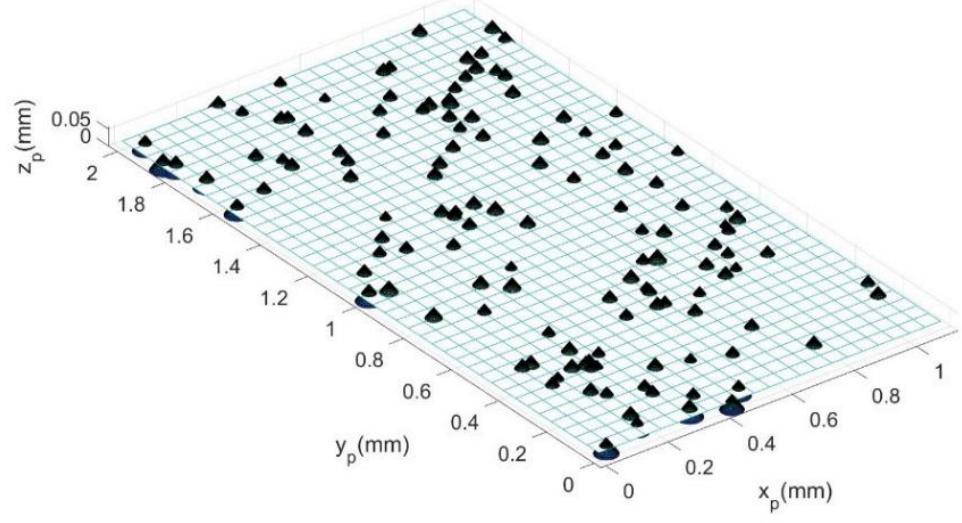

Fig.3 The model of diamond wire saw morphology

In order to express the irregular distribution of abrasive particles on the wire saw, a wire saw coordinate system $o_{c} x_{c} y_{c} z_{c}$ is established as shown in Fig.4. For a random abrasive particle $i$, the position angle on the cross section of the wire saw is $\theta_{i}$, the height from the bottom of the abrasive particle to the surface of the wire saw base is $h_{g i}$ and the distance to the end face of the wire saw is $y_{i}$. The coordinates of the abrasive center (the center of the bottom circle) are $\left(x_{c i}, y_{c i}, z_{c i}\right)$ in the wire saw coordinate system $o_{c} x_{c} y_{c} z_{c}$. It is easy to get the coordinate value by Eq. (7).

$$
\left\{\begin{array}{l}
x_{c i}=\left(\frac{D_{B}}{2}+h_{g i}\right) \cos \theta_{i} \\
y_{c i}=y_{i} \\
z_{c i}=\left(\frac{D_{B}}{2}+h_{g i}\right) \sin \theta_{i}
\end{array}\right.
$$

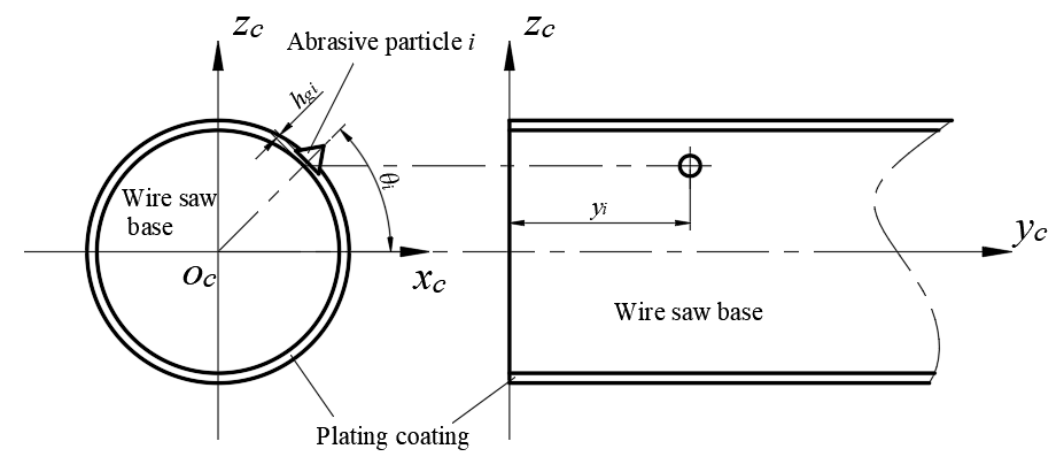


Fig.4 The position of abrasive particle $i$ in the wire saw coordinate system

The position coordinates of abrasive particles in the generated wire saw tool morphology can be converted, that is, the equivalent wire saw coordinate system $o_{p} x_{p} y_{p} z_{p}$ (the coordinate system established on the expansion plane of the wire saw base surface) could be mapped to wire saw coordinate systemo ${ }_{c} x_{c} y_{c} z_{c}$. As shown in Fig.5, the $x_{p}$ axis, $y_{p}$ axis and $y_{p}$ axis of the equivalent wire saw coordinate system represents the circumferential direction of the wire saw, the axial direction of the wire saw, and the direction of the coating thickness of the wire saw respectively. The position coordinates of abrasive particle $i$ in the equivalent wire saw coordinate system are $\left(x_{p i}, y_{p i}, z_{p i}\right)$.

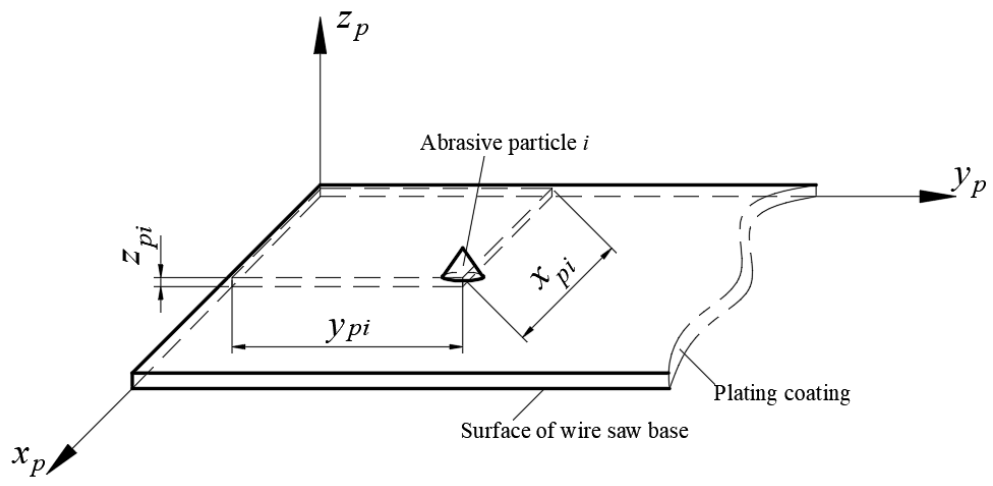

Fig.5 The position of abrasive particle $i$ in the equivalent wire saw coordinate system

The coordinate value of abrasive particle $i$ in the equivalent wire saw coordinate system can be calculated by the following Eq. (8):

$$
\left\{\begin{array}{l}
x_{p i}=\frac{D_{B}}{2} \theta_{i}=\frac{D_{B}}{2} \cdot \arccos \left(\frac{2 x_{c i}}{D_{B}+h_{g i}}\right) \\
y_{p i}=y_{i}=y_{c i} \\
z_{p i}=h_{g i}
\end{array}\right.
$$

Based on the generated wire saw tool surface model, the coordinate value in the wire saw coordinate system of any abrasive particle can be calculated according to Eq. (7-8), which can be used in the later wafer surface morphology prediction modeling work.

\section{The trajectory equation of abrasive particles on the wire saw}

The formation process of wafer surface morphology is the process of interference between abrasive particles with a certain shape and the surface of the workpiece. Therefore, after obtaining the wire saw tool surface model, the trajectory of abrasive particles on the wire saw should be studied to determine the process of material removal formed by the interference between abrasive particles and the workpiece. UAWS slicing is a kind of composite machining technology which applies high frequency harmonic vibration to the wire along the feed direction on the basis of traditional wire saw slicing. The trajectory of abrasive particle movement in UAWS slicing is different from that in traditional wire saw slicing, and it has different effects on the formation of workpiece surface morphology. So, it is necessary to use mathematical formula to express the motion track of abrasive particles on the wire saw in UAWS slicing.

\subsection{The trajectory equation of abrasive particle in workpiece coordinate system}

Since the workpiece does rotation movement in the ground coordinate system, the track 
equation of abrasive particle in the machine tool coordinate system (ground coordinate system) is be derived first. And the track equation of abrasive particle in the workpiece coordinate system can be obtained according the coordinate transformation rule. As shown in Fig.6, the origin $o_{w}$ of the workpiece coordinate system $o_{w} x_{w} y_{w} z_{w}$ coincides with the origin $o$ of the machine tool coordinate system $o_{g} x_{g} y_{g} z_{g}$, and the workpiece coordinate system rotates around the origin $o_{g}$ at a constant rotation speed of $n_{w}$.

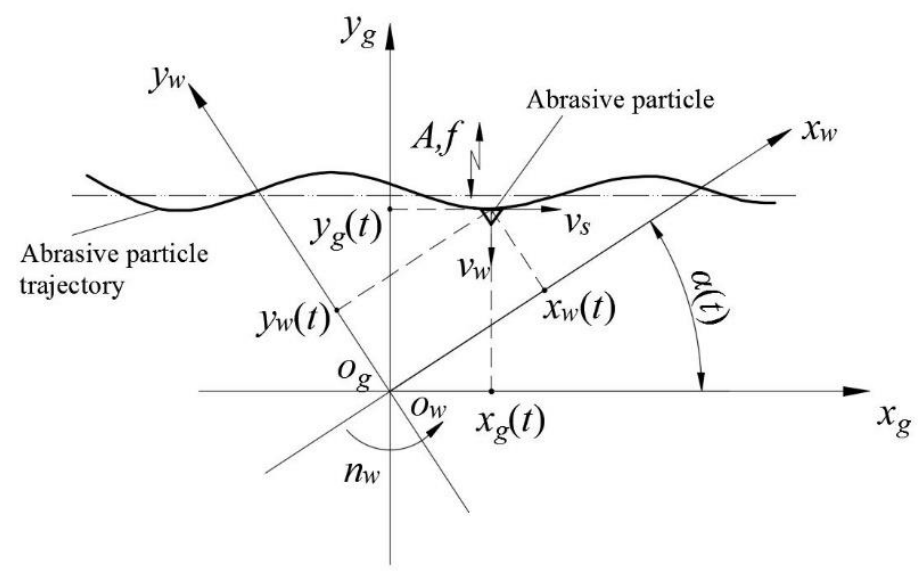

Fig.6 Schematic of abrasive particle trajectory coordinate transformation

As shown in the figure, the motion equation of each motion component of the abrasive particle are shown in Eqs. (9-11):

(I) the axial motion equation with the wire saw:

$$
x_{g}(t)=\left(v_{s} \pm \frac{\pi D_{B} n_{w}}{60}\right) t
$$

(II) the downward feed motion equation with the wire saw:

$$
y_{g}(t)=v_{w} t
$$

(III) the ultrasonic vibration equation with the wire saw:

$$
y_{g}(t)=A \sin (2 \pi f t)
$$

According to the motion synthesis law, the motion trajectory equation of the abrasive particles in the machine tool coordinate system can be obtained:

$$
\left\{\begin{array}{l}
x_{g}(t)=\left(v_{s} \pm \frac{\pi D_{B} n_{w}}{60}\right) t \\
y_{g}(t)=v_{w} t+A \sin (2 \pi f t)
\end{array}\right.
$$

The coordinate value $\left[x_{g}(t), y_{g}(t)\right]$ of abrasive particles in the machine tool coordinate system at any time $t$ can be calculated from Eq. (12). At this time, there is an angular displacement difference $\alpha(t)$ between the workpiece coordinate system and the machine tool coordinate system. The coordinates $\left[x_{w}(t), y_{w}(t)\right]$ of the abrasive particle in the workpiece coordinate system can be obtained by two-dimensional rotation transformation matrix operation:

$$
\left[\begin{array}{l}
x_{w}(t) \\
y_{w}(t)
\end{array}\right]=\left[\begin{array}{cc}
\cos \alpha & \sin \alpha \\
-\sin \alpha & \cos \alpha
\end{array}\right]\left[\begin{array}{l}
x_{g}(t) \\
y_{g}(t)
\end{array}\right]
$$

The coordinates of the abrasive particle in the workpiece coordinate system at time $t$ can be obtained by substituting Eq. (12) into Eq. (13):

$$
\left\{\begin{array}{l}
x_{w}(t)=\left(v_{s} \pm \frac{\pi D_{B} n_{w}}{60}\right) t \cos \alpha+\left[v_{w} t+A \sin (2 \pi f t)\right] \sin \alpha \\
y_{w}(t)=-\left(v_{s} \pm \frac{\pi D_{B} n_{w}}{60}\right) t \sin \alpha+\left[v_{w} t+A \sin (2 \pi f t)\right] \cos \alpha
\end{array}\right.
$$


The angle $\alpha$ in Fig. 6 is acute, but Eq. (13) is derived from the basic definition of trigonometric function, so it is tenable when the angle $\alpha$ is taken to any value, that is Eq. (14) is valid when the workpiece is rotated to any angle during slicing. Because $\alpha$ is a function of $t$, which means that Eq. (14) is tenable at any time $t$. Assuming that when $t=0, x_{w}$ axis and $x_{g}$ axis overlap, the expression of $\alpha$ is:

$$
\alpha(t)=\frac{\pi}{30} n_{w} t
$$

The motion trajectory equation of abrasive particles on the wire saw surface in the workpiece coordinate system during the UAWS slicing process can be obtained by substituting Eq. (15) into Eq. (14):

$$
\left\{\begin{array}{l}
x_{w}(t)=\left(v_{s} \pm \frac{\pi D_{B} n_{w}}{60}\right) t \cos \left(\frac{\pi}{30} n_{w} t\right)+\left[v_{w} t+A \sin (2 \pi f t)\right] \sin \left(\frac{\pi}{30} n_{w} t\right) \\
y_{w}(t)=-\left(v_{s} \pm \frac{\pi D_{B} n_{w}}{60}\right) t \sin \left(\frac{\pi}{30} n_{w} t\right)+\left[v_{w} t+A \sin (2 \pi f t)\right] \cos \left(\frac{\pi}{30} n_{w} t\right)
\end{array}\right.
$$

\subsection{Establishment of the workpiece surface morphology coordinate system}

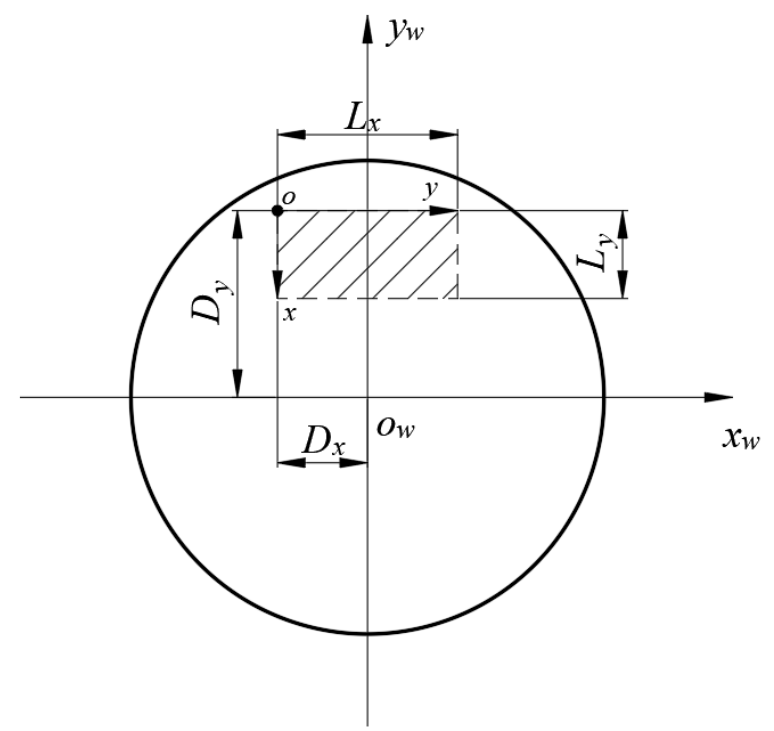

Fig.7 Schematic of surface morphology modeling area

During the modeling of the workpiece surface morphology, the selected area of modeling area is much smaller than the actual sliced wafer surface area in order to study the micro-morphology of workpiece surface. An area is selected on the wafer surface for modeling, and the relative position of the workpiece surface morphology modeling area in the workpiece coordinate system $o_{w} x_{w} y_{w}$ is shown in Fig.7. The length and width of the modeling area are $L_{x}$ and $L_{y}$. Meanwhile, the workpiece surface morphology coordinate system oxyz is established and the coordinate of origin $o$ relative to the origin $o_{w}$ of the workpiece coordinate system is $\left(D_{x}, D_{y}\right)$. The size and location of the modeling area can be changed by changing the values of $L_{x}, L_{y}, D_{x}$ and $D_{y}$, making the model is universal for the entire wafer surface.

For convenience, the modeling area was rotated $90^{\circ}$. As shown in Fig.8, the workpiece surface morphology coordinate system is established, and the origin $o$ is set at $0.5 D_{B}$ away from the center of the cross section of the wire saw. 


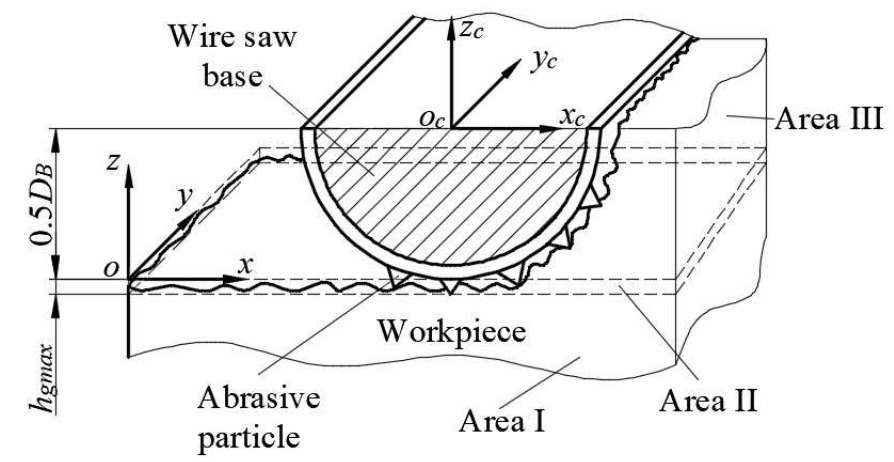

Fig.8 Schematic of workpiece surface morphology coordinate system

Because of the symmetry of the wire saw, half of the cross section of the wire saw was selected for analysis. During the slicing process, the wire saw is moved along the $y$ axis direction and along the $x$ direction. Since the wire saw surface is filled with abrasive particles, the workpiece materials in the projection area of the wire saw base along the feed direction, namely area III, are bound to be removed, and its thickness is exactly the diameter $D_{B}$ of the wire saw base. The maximum protruding height of abrasive particle on the wire saw surface is set as $h_{\text {gmax }}$, the workpiece area with the maximum extension of $h_{\text {gmax }}$ from the edge of area III during the sire saw slicing process is defined as area II. The workpiece materials in this area II may be removed or may not be removed which cause the uneven surface after sliced and this is the final wafer surface we will study. The remaining area I will not be removed because the abrasive particles have no contact with the workpiece.

\subsection{The trajectory equation of abrasive particles in the workpiece surface morphology coordinate system}

The trajectory equation of abrasive particles in the workpiece coordinate system has been obtained. And then the trajectory equation of abrasive particles in the workpiece surface morphology coordinate system can be deduced. The slicing starting time is set to $t_{0}=0 \mathrm{~s}$, and the distance from the origin $o_{w}$ is $D_{0}$, and the rotation angle of the workpiece coordinate system relative to the machine tool coordinate system is $\alpha_{0}$ at this time. As shown in Eq. (17), The trajectory equation of the origin $o_{c}$ of the wire saw coordinate system in the workpiece coordinate system can be easily obtained.

$$
\left\{\begin{array}{l}
x_{w o}(t)=\left(v_{s} \pm \frac{\pi D_{B} n_{w}}{60}\right) t \cos \alpha_{0}+\left[v_{w} t+A \sin (2 \pi f t)\right] \sin \alpha_{0} \\
y_{w o}(t)=-\left(v_{s} \pm \frac{\pi D_{B} n_{w}}{60}\right) t \sin \alpha_{0}+\left[v_{w} t+A \sin (2 \pi f t)\right] \cos \alpha_{0}+D_{0}
\end{array}\right.
$$

According to the relationship between the workpiece coordinate system and the workpiece surface morphology coordinate system in the modeling area, the trajectory equation of the origin $o^{\prime}$ of the wire saw coordinate system in the workpiece surface morphology coordinate system can be obtained:

$$
\left\{\begin{array}{l}
x(t)=\left(v_{s} \pm \frac{\pi D_{B} n_{w}}{60}\right) t \sin \alpha_{0}-\left[v_{w} t+A \sin (2 \pi f t)\right] \cos \alpha_{0}-D_{0}+D_{x} \\
y(t)=\left(v_{s} \pm \frac{\pi D_{B} n_{w}}{60}\right) t \cos \alpha_{0}+\left[v_{w} t+A \sin (2 \pi f t)\right] \sin \alpha_{0}+D_{y} \\
z(t)=0.5 D_{B}
\end{array}\right.
$$

Where, the diameter of wire saw base is $D_{B}, D_{x}$ and $D_{y}$ are the coordinate values of the origin $o$ with respect to the workpiece coordinate system $o_{w}$, as shown in Fig.7.

For any abrasive particle $i$ on the wire saw surface, the time $t_{i}$ when it reaches the edge of the 
modeling area is $t_{i}-t_{0}$ later than the starting time $t_{0}$. Then the trajectory equation of abrasive particle $i$ in the workpiece surface morphology coordinate system can be obtained by Eq. (19).

$$
\left\{\begin{array}{l}
x(t)=\left[\left(v_{s} \pm \frac{\pi D_{B} n_{w}}{60}\right)\left(t-t_{i}\right)-D_{x}\right] \sin \alpha_{i}-\left[D_{y}-v_{w}\left(t-t_{i}\right)-A \sin 2 \pi f\left(t-t_{i}\right)\right] \cos \alpha_{i}+D_{y} \\
y(t)=\left[\left(v_{s} \pm \frac{\pi D_{B} n_{w}}{60}\right)\left(t-t_{i}\right)-D_{x}\right] \cos \alpha_{i}+\left[D_{y}-v_{w}\left(t-t_{i}\right)-A \sin \left(2 \pi f\left(t-t_{i}\right)\right)\right] \sin \alpha_{i}+D_{x} \\
z(t)=0.5 D_{B}+\left(0.5 D_{B}+h_{g i}\right) \sin \theta_{i}
\end{array}\right.
$$

Where, the height from the bottom of the abrasive particle to the surface of the wire saw base is $h_{g i}$, the time $t_{i}$ when abrasive particle $i$ reaches the edge of the modeling area can be calculated according to the position of the abrasive particle relative to the origin $o_{c}$ of the wire saw coordinate system:

$$
t_{i}=\frac{y_{c i}}{v_{s}}
$$

And at time $t_{i}$, the rotation angle of the workpiece coordinate system with respect to the machine tool coordinate system can be obtained by the following Eq. (21).

$$
\alpha_{i}=\alpha_{0}+\frac{\pi}{30} n_{w} t_{i}
$$

Eq. (19) is the trajectory equation of any abrasive particles on the wire saw surface during the UAWS slicing in the determined workpiece surface morphology coordinate system oxyz and it is universal.

\section{Prediction model of wafer surface morphology}

\subsection{Mixed material removal model in wire saw slicing single crystal silicon}

Ductile or brittle removal may occur in grinding hard and brittle materials, such as single crystal silicon, depending on the load applied on the abrasive particles [5]. As shown in the Fig.9(a), when the abrasive particle is subjected to a small load, which is lower than the critical load, ductile removal occurs and the cross section of material removed by abrasive particle is the abrasive cross section. As shown in the Fig.9(b), brittle removal occurs when the load on the abrasive particles is greater than the critical load. At this point, transverse crack and median crack will be generated below the abrasive particle and after the abrasive particle scratch across the workpiece surface, transverse crack will extend to the workpiece surface with the abrasive particles leaving, forming chips and causing material removal. The removed area is the one formed by the common envelope of transverse crack and workpiece surface. The depth $C_{h}$ and width $C_{l}$ of transverse crack can be calculated by Eqs. (22-23) [16-17].

$$
\begin{gathered}
C_{h}=0.43 \frac{\sqrt{\sin \beta \cdot E \cdot F_{n g}}}{H} \cdot \sqrt[3]{\cot \beta} \\
C_{l}=0.226 \sqrt[8]{\frac{E^{3} \cdot\left(1.565 F_{n g}\right)^{5}}{\left(0.3 K_{I C} H\right)^{4}}}
\end{gathered}
$$

Where $\beta$ is half of the top angle of abrasive, $E$ is the Young's modulus, $F_{n g}$ is the normal resultant force of an abrasive, $H$ is the Vickers hardness, $K_{I C}$ is the fracture toughness. 


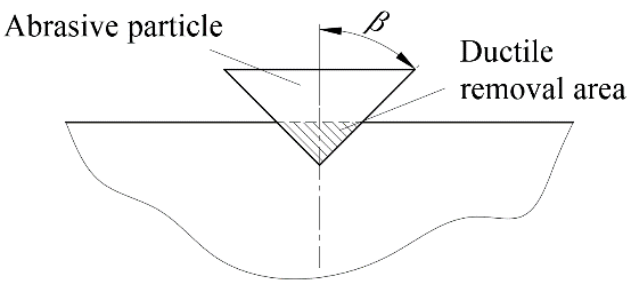

(a) Ductile removal model

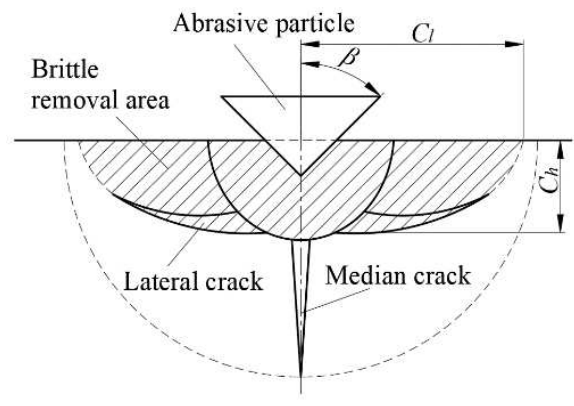

(b) Brittle removal model

Fig.9 Schematic of mixed material removal model in single crystal silicon

In order to introduce the mixed material removal mode into the wafer surface morphology prediction model, the model of the workpiece material removed in Fig.9 is simplified to the brittle and plastic mixed removal model as shown in Fig.10.

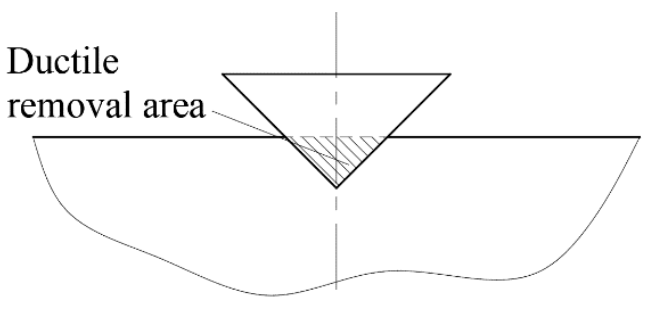

(a) Simplified ductile removal model

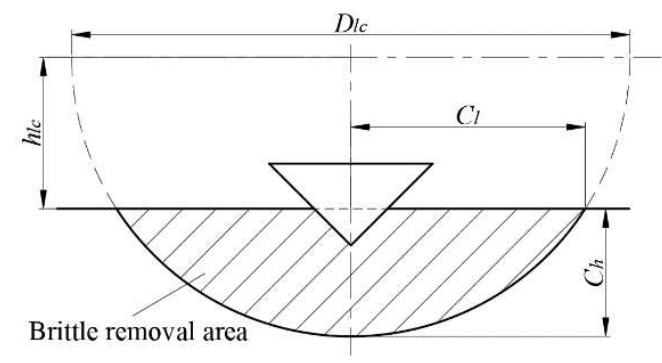

(b) Simplified brittle removal model

Fig.10 Schematic of simplified mixed material removal model

The section shape of the area removed by the abrasive particle cutting in ductile removal mode is triangular [18], and its size is related to the cutting depth of abrasive particles. The shape of the area removed by the abrasive particle in brittle removal mode is approximately a spherical crown, whose diameter $D_{l c}$ and the height of center $h_{l c}$ depend on the depth and width of the transverse crack, which can be obtained by the following Eq. (24).

$$
\left\{\begin{array}{l}
D_{l c}=\frac{C_{h}{ }^{2}+C_{l}{ }^{2}}{C_{h}} \\
h_{l c}=\frac{C_{l}{ }^{2}-C_{h}{ }^{2}}{2 C_{h}}
\end{array}\right.
$$

When the wire saw feeds downward to slice the workpiece, the abrasive particles at different positions on the cross section of the wire saw are subjected to different loads, resulting in different material removal modes in Fig.11.

The abrasive particles near the bottom of the slicing groove impact the workpiece under ultrasonic excitation and cut the workpiece with a relatively large depth. At this time, the abrasive particle is subjected to a large load, resulting in the brittle removal of single crystal silicon. However, the cut depth of the abrasive particles on the side of the groove is small which means that the load on the abrasive particles is small, and abrasive particles only produce ductile removal on the wafer surface. In previous studies [19-20], the direct influence of ductile removal of abrasive particles on the side of the groove on the wafer surface formation process when modeling the workpiece surface morphology during wire saw slicing was generally considered, while the indirect influence of brittle removal of abrasive particles close the bottom of the groove was ignored 


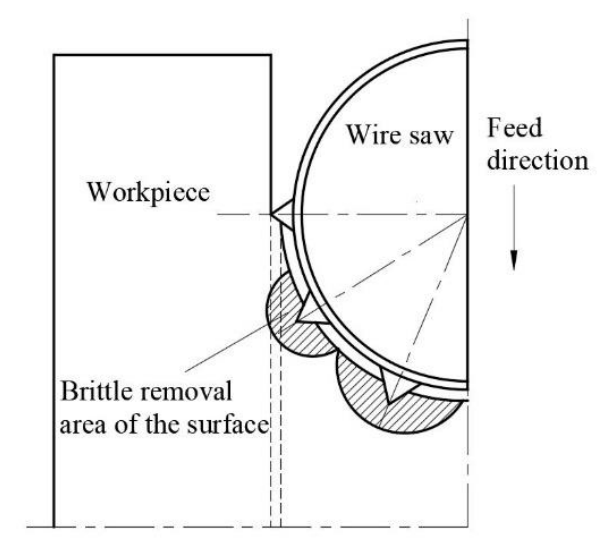

Fig.11 Schematic of wafer surface formation in mixed material removal mode

\subsection{Prediction model of wafer surface morphology in UAWS slicing single crystal silicon}

In the method of the of workpiece surface morphology prediction [21], the topological matrix $g_{m n}$ is usually used to represent the workpiece surface morphology. The grid is divided according to spacing $\Delta x$ in the $x$ direction and spacing $\Delta y$ in the $y$ direction on the surface of the workpiece. The height value $z(m, n)$ at grid point $P(m, n)$ is taken as the element in the workpiece topological matrix $g_{m n}$, as shown in Fig.12.

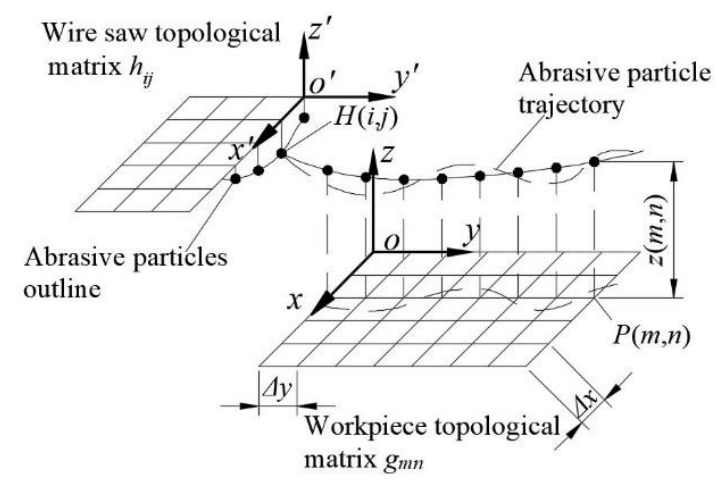

Fig.12 Schematic of workpiece topology matrix

The general calculation method of workpiece surface morphology [22-23] requires static sampling of surface morphology of grinding wheel, wire saw and other tools, which is represented by topological matrix $h_{i j}$. As shown in Fig.12, the height value $h(i, j)$ of the sampling point $H(i, j)$ on the wire saw surface contour is taken as the element of that point in the topological matrix $h_{i j}$. During calculation, the sampling points in the topological matrix of the wire saw are taken out in turn and the trajectory of the abrasive particle in that sampling point is calculated. Then, the height value of each grid point $P(m, n)$ through which the trajectory passes of the workpiece topological matrix, and after all the trajectories passing through the grid point $P(m, n)$ were calculated, the minimum height value $\min (z(m, n))$ is the final residual height of the workpiece surface at this point after processing. The workpiece surface morphology can be obtained when the residual height value of all the lattice points on the workpiece surface were calculated. However, this method is no longer applicable in UAWS slicing because the trajectory of abrasive particles in this process is different. At this time, the trajectory of the sampling point $H(i, j)$ in the wire saw surface contour is similar to sine curve, so the projection of the trajectory on the xoy plane cannot completely cover the lattice points on the workpiece surface and that is impossible to calculate the height value 
corresponding to the trajectory of the sampling point $H(i, j)$ of the abrasive particle contour at the lattice point $P(m, n)$ of the workpiece topological matrix. This is because the wire saw topological matrix is used to represent the surface morphology of the wire saw, which means that static sampling is carried out for the wire saw abrasive particles contour in advance. When the ultrasonic vibration is applied, the sampling points of the wire saw abrasive particles contour cannot always be aligned with the lattice points of the workpiece surface. Dynamic profile sampling method ${ }^{[16]}$ can used to solve this problem. Dynamic sampling of abrasive particles contour can be conducted following the ultrasonic vibration in this method, so as to ensure that the residual height value of workpiece surface at the lattice point $P(m, n)$ can be calculated.

Firstly, a series of isometric sampling sections parallel to the yoz plane is set in the selected workpiece surface morphology modeling area, and these sampling sections coincide with the lattice points of the workpiece topological matrix and are numbered as 1, 2, 3, $4 \ldots$ in the positive direction of $y$ axis from the origin $o$ as shown in Fig.13.

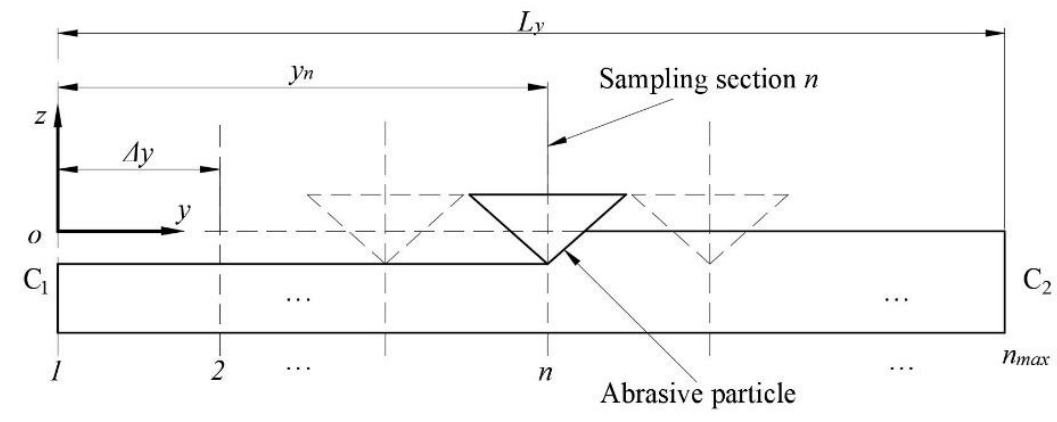

Fig.13 Sampling sections setting

During slicing, a certain abrasive particle enters the modeling area from point $\mathrm{C} 1$ and leaves the modeling area at point $\mathrm{C} 2$. The sampling section 1 which coincides with point $\mathrm{C} 1$ is the first sampling section where abrasive particle interferes with the workpiece, and the sampling cross section $n_{\max }$ to the left of $\mathrm{C} 2$ is the last one. That is, abrasive particles successively pass through the sampling section starting from 1 to $n_{\max }$. The workpiece surface morphology after the abrasive particles cut the workpiece can be obtained by calculating the residual height value of all lattice points in these sections. The length of the abrasive particle grinding through the workpiece surface is just the length $L_{y}$ of the workpiece surface morphology modeling area, so the value of $n_{\max }$ can be calculated:

$$
n_{\text {max }}=\left[\frac{L_{y}}{\Delta y}\right]
$$

When calculating the residual height value of each lattice point in the sampling section $n$, the horizontal distance $y_{n}$ from the section to the origin $o$ of the workpiece surface morphology coordinate system should be calculated first, as shown in the following Eq. (26).

$$
y_{n}=(n-1) \Delta y
$$

The horizontal distance $x_{n}$ and vertical distance $z_{n}$ from the abrasive particle in the sampling section to the origin $o$ of the workpiece surface morphology coordinate system can be inversely solved by substituting $y_{n}$ into Eq. (19). In this way, the position of abrasive particle in the sampling section $n$ can be determined. Then, sampling points are set in the sampling section, as shown in Fig.14. Due to the symmetry of abrasive particles, the equation of abrasive contour can be expressed by Eq. (27): 


$$
z=\left\{\begin{aligned}
-1.43\left(x-x_{n}+0.7 d_{g}\right)+z_{n}, & x \leq x_{n} \\
1.43\left(x-x_{n}-0.7 d_{g}\right)+z_{n}, & x>x_{n}
\end{aligned}\right.
$$

Where $d_{g}$ is the size of abrasive particles, which can be obtained from the surface model of the wire saw.

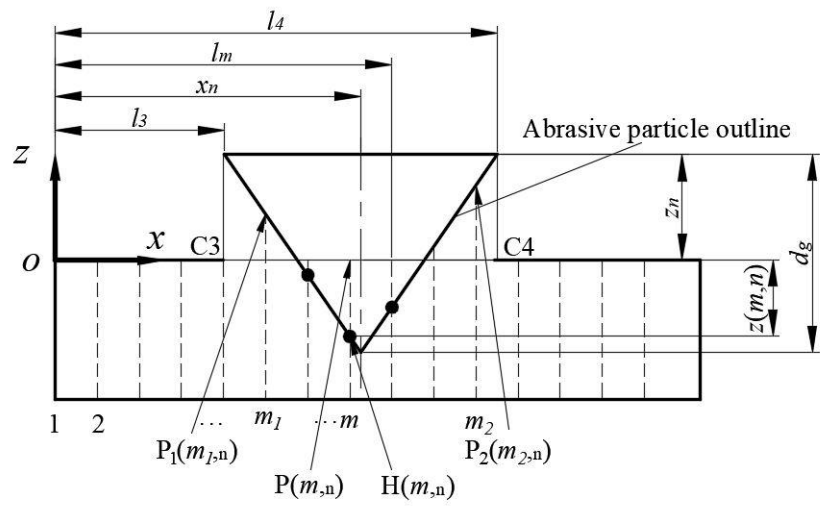

Fig.14 Sampling points setting in the sampling section $n$

In the Fig. 14, C3 and C4 are set as the first and last sampling points respectively. By calculating the sampling points between $\mathrm{C} 3$ and $\mathrm{C} 4$, the corresponding height value of each sampling point on the abrasive contour can be obtained. The first sampling point $\mathrm{P}_{1}\left(m_{1}, n\right)$ on the right of $\mathrm{C} 3$ is the first sampling point, and $\mathrm{P}_{2}\left(m_{2}, n\right)$ on the left of $\mathrm{C} 4$ is the last sampling point. Values of $m_{1}$ and $m_{2}$ can be obtained by the following Eq. (28).

$$
\left\{\begin{array}{l}
m_{1}=\left[\frac{l_{3}}{\Delta x}\right]+2=\left[\frac{x_{n}-0.7 d_{g}}{\Delta x}\right]+2 \\
m_{2}=\left[\frac{l_{4}}{\Delta x}\right]+1=\left[\frac{x_{n}+0.7 d_{g}}{\Delta x}\right]+1
\end{array}\right.
$$

Then, all sampling points from $\mathrm{P}_{1}$ to $\mathrm{P}_{2}$ are sampled. The horizontal distance from a sampling point $H(m, n)$ to the origin $o$ of the workpiece surface morphology coordinate system is $l_{m}$, which can be calculated by Eq. (29).

$$
l_{m}=(m-1) \Delta x
$$

By substituting $l_{m}$ into the abrasive particle contour equation, the vertical value $\mathrm{z}(m, n)$ of the sampling point can be obtained. When the height value $\mathrm{z}(m, n)$ is smaller than the original height value of the grid point $P(m, n)$, the abrasive particle is considered to interfere with the workpiece and it is assumed that the interfering material is completely removed, then the height value of the sampling point $H(m, n)$ is assigned to the grid point $P(m, n)$ to complete the update of the height value of the grid point. Similarly, after updating all lattice points in the sampling section, the calculation in the sampling section $\mathrm{n}$ is completed. After all sampling sections numbered from 1 to $n_{\max }$ are updated, the workpiece surface morphology after abrasive particles grinding can be obtained. On this basis, sampling, calculation and updating of all abrasive particles in the surface model of the wire saw can be conducted to obtain the workpiece surface morphology after wire saw slicing.

The above method is only a general description of the whole modeling process in principle. In order to truly reflect the processing of UAWS slicing single crystal silicon and make the model more accurate, the mixed material removal model is introduced into the calculation process of dynamic contour sampling method. As shown in Fig.15, different abrasive particles on the cross section of the wire saw have different position angles, and the relationship between them and the workpiece surface morphology coordinate system is also changed. At this time, the strategy of sampling point 
layout within the sampling section $n$ needs to be changed and the actual interference between abrasive particles and workpiece should be analyzed again in the sampling section with serial number $n$.

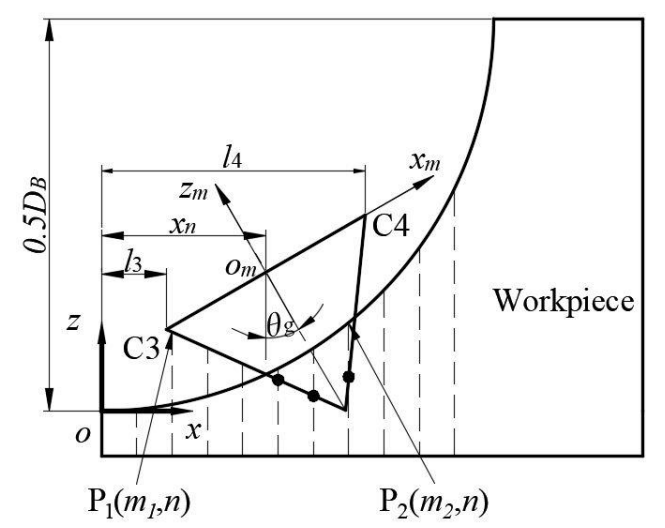

Fig.15 Improved sampling points setting in the sampling section $n$

During modeling, only the abrasive particles in the $1 / 4$ cross section of wire saw were used for analysis due to the symmetry of the wire saw. The area to be modeled is changed from the previous plane to the circular arc, and the radius of the circular arc is the radius of the wire saw matrix. Because this part will be removed in the process of slicing, its influence on workpiece surface when modeling is not considered. Meanwhile, only the influence of brittle removal mode of abrasive particles close to the side of groove on the final surface of the workpiece was considered, that is, only the abrasive particles whose position angle $\theta_{i}$ is in the range of $[3 \pi / 2,7 \pi / 4]$ on the cross section of wire saw are calculated. As shown in Fig.16, a certain abrasive particle on the cross section of the wire saw interferes with the circular surface. The equation of abrasive particle profile in the abrasive coordinate system $o_{m} x_{m} z_{m}$ is:

$$
z_{m}=\left\{\begin{array}{r}
-1.43\left(x+0.7 d_{g}\right)-d_{g}, x \leq 0 \\
1.43\left(x-0.7 d_{g}\right)+d_{g}, x>0
\end{array}\right.
$$

And the relationship between the abrasive coordinate system and the coordinate system oxyz exists as follows:

$$
\left[\begin{array}{l}
x_{m} \\
z_{m}
\end{array}\right]=\left[\begin{array}{cc}
\cos \theta_{g} & \sin \theta_{g} \\
-\sin \theta_{g} & \cos \theta_{g}
\end{array}\right]\left[\begin{array}{l}
x-x_{n} \\
z-z_{n}
\end{array}\right]
$$

Then the equation of abrasive particle profile in the workpiece surface morphology coordinate system oxyz can be obtained.

$$
z=\left\{\begin{array}{l}
\frac{0.7 \sin \theta_{g}-\cos \theta_{g}}{\sin \theta_{g}+0.7 \cos \theta_{g}}\left(x-x_{n}-d_{g} \sin \theta_{g}\right)+z_{n}-d_{g} \cos \theta_{g}, x \leq x_{n} \\
\frac{0.7 \sin \theta_{g}+\cos \theta_{g}}{0.7 \cos \theta_{g}-\sin \theta_{g}}\left(x-x_{n}-d_{g} \sin \theta_{g}\right)+z_{n}-d_{g} \cos \theta_{g}, x>x_{n}
\end{array}\right.
$$

Similarly, sampling points are set from the center of the abrasive particles to both sides and $m_{1}$ and $m_{2}$ in the sampling points $\mathrm{P}_{1}\left(m_{1}, n\right)$ and $\mathrm{P}_{2}\left(m_{2}, n\right)$ can be obtained.

$$
\left\{\begin{array}{l}
m_{1}=\left[\frac{l_{3}}{\Delta x}\right]+2=\left[\frac{x_{n}-0.7 d_{g} \cos \theta_{g}}{\Delta x}\right]+2 \\
m_{2}=\left[\frac{l_{4}}{\Delta x}\right]+1=\left[\frac{x_{n}+0.7 d_{g} \cos \theta_{g}}{\Delta x}\right]+1
\end{array}\right.
$$

Where $\theta_{g}$ is the angle between the center line of the abrasive particle and the vertical direction, and $\theta_{g}=\theta_{i}-3 \pi / 2$.

As shown in Fig.16, The workpiece surface during processing is not an ideally smooth surface, 
but a bumpy surface lower than the initial circular arc. There are a series of interference points from $H_{1}\left(m_{1}, n\right)$ to $H_{2}\left(m_{2}, n\right)$, and each sampling point has different cutting depth.

In order to judge whether brittle removal occurs at this point, the normal load on the abrasive particles at this position should be calculated first, and then compared with the critical load of single crystal silicon $(26 \mathrm{mN})$ [24]. When the normal load is greater than the critical load, brittle fracture occurs to the material at this point. On this basis, the abrasive particle interference is calculated in each sampling section, and if brittle fracture occurs, the abrasive particle contour is changed to the brittle removal contour in the mixed removal model for sampling calculation. Because those sampling points where interference occurs in the sampling section $n$ have different cutting depth, and the surface during processing is not smooth but the height difference between adjacent lattice is not big. At this time, the average cutting depth $d_{\text {pave }}$ of this abrasive particle can be calculated according to the cutting depth of each lattice $d_{p}$, then the normal load of abrasive particle can be obtained by substituting $d_{\text {pave }}$ into the formula of normal sawing force of single abrasive particle. The average cutting depth $d_{\text {pave }}$ can be calculated by Eq. (34).

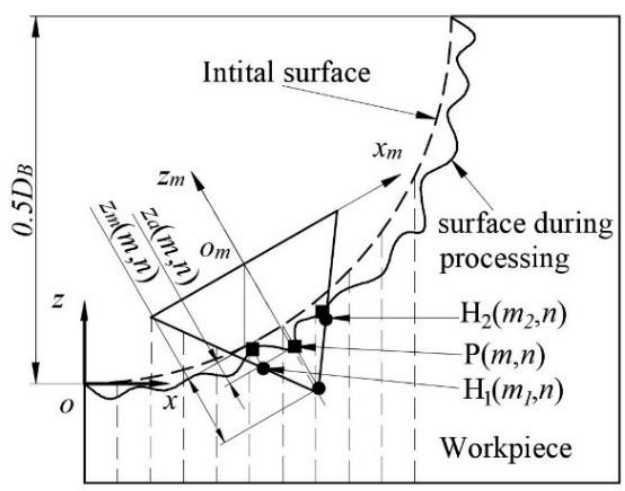

Fig.16 Schematic of actual interference situation during processing

$$
d_{\text {pave }}=\frac{\sum_{m_{1}}^{m_{2}}\left[z_{m}(m, n)-z_{a}(m, n)\right]}{m_{2}-m_{1}+1}
$$

Where $z_{m}(m, n)$ is the ordinate value of sampling point $\mathrm{P}(m, n)$ in the abrasive particle coordinate system $o_{m} x_{m} z_{m}$, and $z_{a}(m, n)$ is the vertical coordinate value of abrasive particle contour corresponding to sampling point $\mathrm{P}(m, n)$ in the abrasive particle coordinate system. In order to facilitate calculation, the corresponding coordinate value of sampling point $\mathrm{P}(m, n)$ in the abrasive particle coordinate system is obtained in advance, and then the cutting depth $d_{p}$ of each lattice point can be calculated.

When brittle fracture is determined to occur in the sampling section with serial number of $n$, the shape of the material removed from the workpiece surface is a spherical crown with a diameter of $D_{l c}$ located in the sampling section $n$, and its spherical coordinates $\left(x_{p m}, z_{p m}\right)$ in the abrasive particle coordinate system can be obtained by the following Eq. (35).

$$
\left\{\begin{array}{c}
x_{p m}=0 \\
z_{p m}=-\left[d_{g}-\left(\sqrt{\left(\frac{D_{l c}}{2}\right)^{2}-\left(\frac{C_{l}}{2}\right)^{2}}+d_{\text {pave }}\right)\right.
\end{array}\right.
$$

According to Eq. (31), the coordinates $\left(x_{p}, z_{p}\right)$ of the spherical center of the brittle removal contour in the workpiece surface morphology coordinate system can be obtained. Then, the equation of the brittle removal contour is shown in Eq. (36). 


$$
\left(x-x_{p}\right)^{2}+\left(z-z_{p}\right)^{2}=\left(\frac{D_{l c}}{2}\right)^{2}
$$

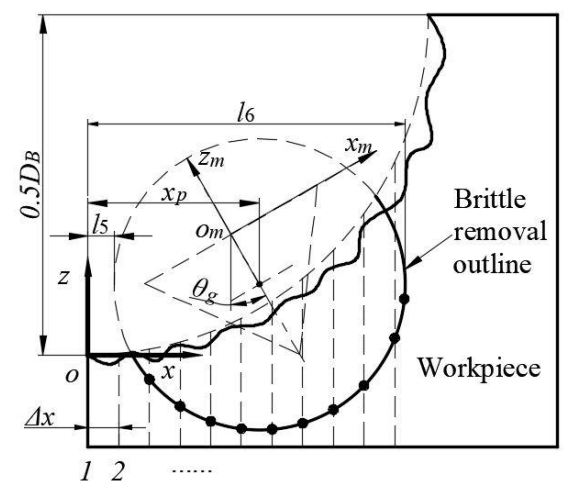

Fig.17 Schematic of brittle removal sampling

By substituting Eq. (36) for Eq. (27) for sampling, the residual height value of the lattice point on the workpiece surface after brittle removal occurs at sampling section $n$ can be obtained. If brittle removal does not occur, Eq. (32) can be used for sampling. Due to the shape of the material being removed in brittle mode is spherical crown, several sampling sections adjacent to sampling cross section $n$ will be affected as shown in Fig.18.

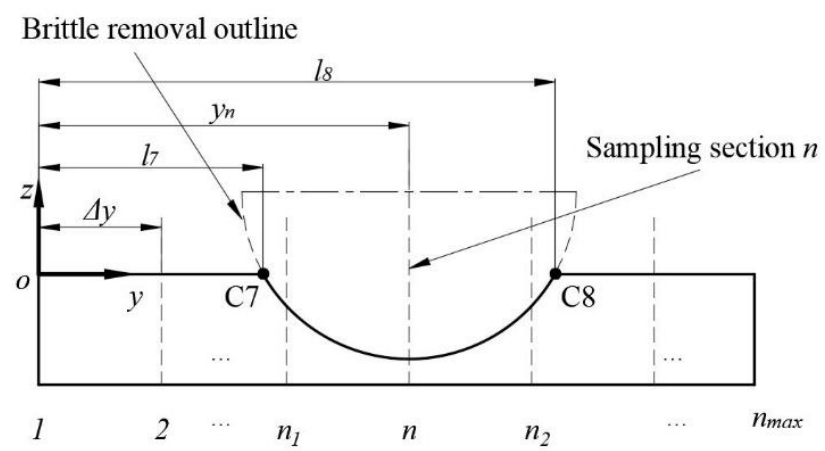

Fig.18 Sampling section distribution affected by brittle removal

The intersection points of brittle removal contour and ideal workpiece surface are $\mathrm{C} 7$ and $\mathrm{C} 8$. The section $n_{1}$ to the right of $\mathrm{C} 7$ is the first affected sampling section, and the section $n_{2}$ to the left of $\mathrm{C} 8$ is the last affected sampling section. That is, the affected sampling sections are a series of sampling sections from $n_{1}$ to $n_{2}$, in which the residual height value of the lattice point on the workpiece surface needs to be recalculated. Similarly, the value of $n_{1}$ and $n_{2}$ can be calculated from $l_{7}$ and $l_{8}$. Within the several sampling sections adjacent to sampling section $n$, the brittle removal contour is still a semicircle with a change in diameter. And for the $(n+k)$ or $(n-k)$ sampling section, the diameter of the brittle removal contour in the sampling section is:

$$
D_{k}=2 \sqrt{\left(\frac{D_{l c}}{2}\right)^{2}-(k \Delta y)^{2}}
$$

Substituting $D_{k}$ for $D_{l c}$ into Eq. (36), the equation of the brittle removal contour in the $(n+k)$ or $(n-k)$ sampling section can be obtained.

$$
\left(x-x_{p}\right)^{2}+\left(z-z_{p}\right)^{2}=\left(\frac{D_{k}}{2}\right)^{2}
$$

Then, sampling is carried out within these sampling sections near sampling section $n$ to update the residual height value of the lattice point on the workpiece surface. After the samplings of the 
brittle removal contour in a series of sampling sections from $n_{1}$ to $n_{2}$ are completed, the brittle removal sampling contour is truly fully reflected in the workpiece surface morphology, simulating the influence of brittle removal of the workpiece.

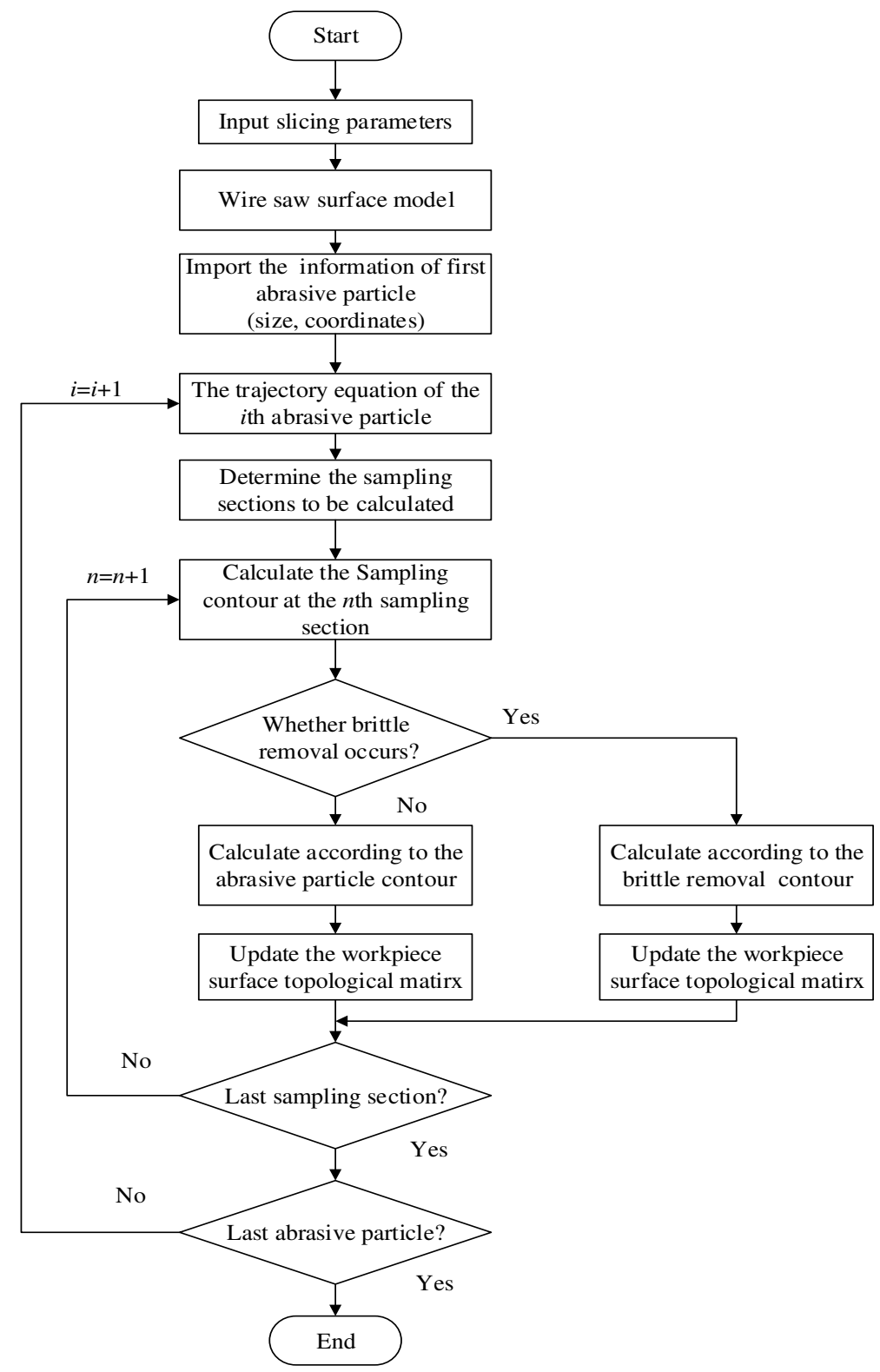

Fig.19 Flow chart of wafer surface morphology prediction

The calculation flow chart of the above model is shown in Fig.19. According to this method, the wafer surface morphology in UAWS slicing single crystal silicon can be predicted.

\section{Verification experiment and results}

The verification experiment was conducted with an improved reciprocating diamond wire saw slicing machine tool and experiment equipment is shown in Fig.20. The workpiece is cylindrical single crystal silicon with a diameter of $36 \mathrm{~mm}$ and a length of $100 \mathrm{~mm}$. The diameter of the wire saw is $0.3 \mathrm{~mm}$ and the average size of the abrasive particles is $60 \mu \mathrm{m}$. The nominal diameter of the wire saw is about $0.35 \mathrm{~mm}$, and the total length of wire saw is $120 \mathrm{~m}$. The tension force of wire saw is set to $112 \mathrm{~N}$ through the machine tool before starting to slice. The working frequency of the 
ultrasonic generating device is $19.8 \sim 20.2 \mathrm{KHz}$, and the vibration frequency is adjusted so that it resonates with the wire saw, thus enabling ultrasound-assisted wire saw processing. The output amplitude of the horn end is $0 \sim 15 \mu \mathrm{m}$ and the DWS vibration displacement sensor is used to monitor the vibration displacement of the wire saw.

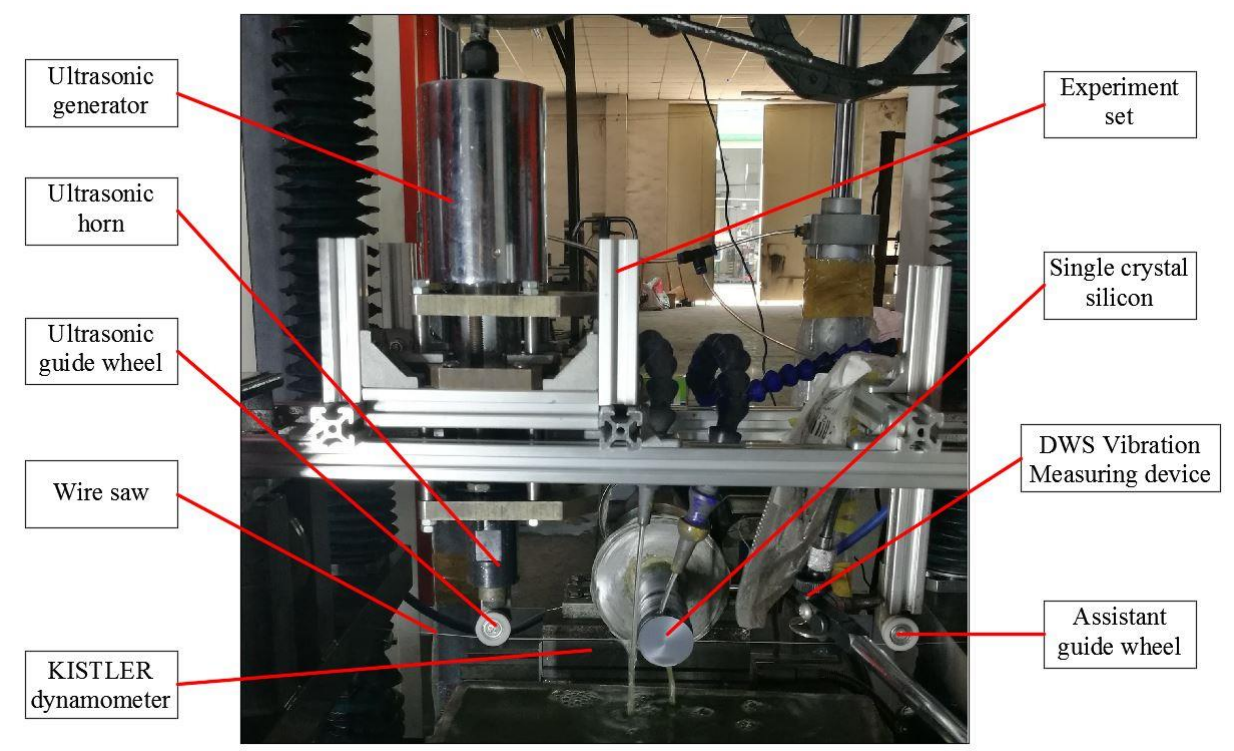

Fig.20 Experiment equipment

The verification experimental parameters were designed as shown in Table 1, and ten sets of experiments were conducted. The ultrasonic vibration amplitude $A=10 \mu \mathrm{m}$, the ultrasonic frequency $f=20 \mathrm{KHz}$. After the experiment, the KEYENCE VXH6000 optical microscope with 2000 magnification was used to observe the surface morphology of the wafer. Besides that, the surface roughness Ra of the wafer was observed by the Mitutoyo SJ-201 surface roughness measuring instrument.

Table 1 Experimental parameters

\begin{tabular}{cccc}
\hline Tests & $v_{s}(\mathrm{~m} / \mathrm{s})$ & $v_{w}(\mathrm{~mm} / \mathrm{min})$ & $n_{w}(\mathrm{r} / \mathrm{min})$ \\
\hline 1 & 2 & 1.0 & 10 \\
2 & 4 & 1.0 & 10 \\
3 & 6 & 1.0 & 10 \\
4 & 8 & 1.0 & 10 \\
5 & 4 & 0.5 & 10 \\
6 & 4 & 1.5 & 10 \\
7 & 4 & 2.0 & 10 \\
8 & 4 & 1.0 & 5 \\
9 & 4 & 1.0 & 15 \\
10 & 4 & 1.0 & 20 \\
\hline
\end{tabular}

The selected modeling area size is $0.15 \mathrm{~mm} \times 0.15 \mathrm{~mm}$. Moreover, the modeling area is symmetrical about the $y_{w}$ axis of the workpiece coordinate system and the horizontal symmetry axis of modeling area is $9 \mathrm{~mm}$ away from the workpiece center. The wafer surface morphology in UAWS slicing single crystal silicon can be predicted according to the prediction process shown in Fig.19. The measured wafer surface morphology of test 7 is shown in Fig.21, and Fig.22 is the predicted wafer surface morphology under the same experimental parameters. 


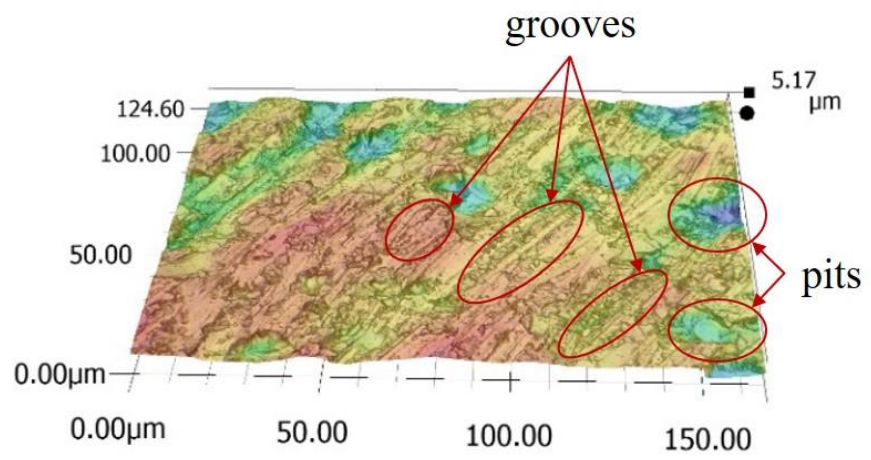

Fig.21 Measured wafer surface morphology of test 7

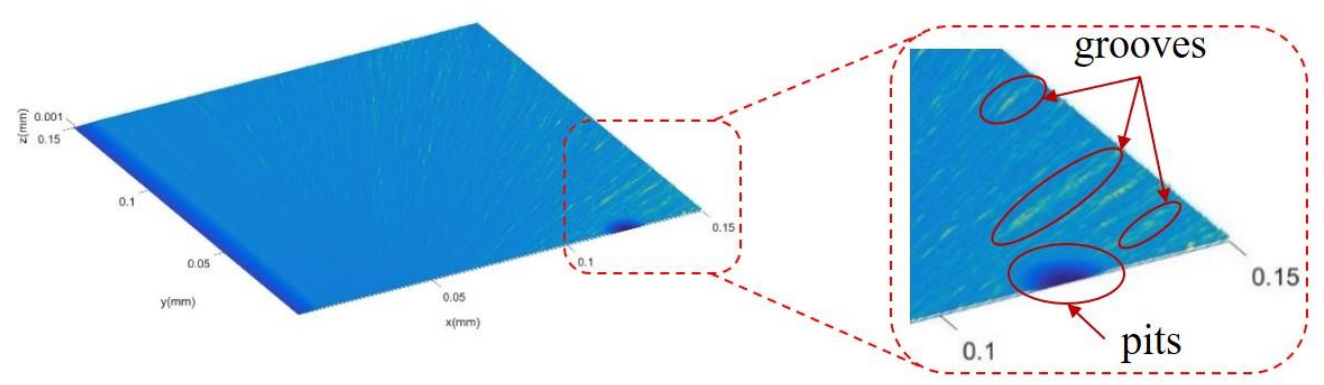

Fig.22 Predicted wafer surface morphology of test 7

According to Fig.21 and Fig.22, it can be seen that the groove direction of the wafer surface in Fig.22 is slightly curved, and the grooves interfere with each other, cutting off the peak on both sides of the groove. At the same time, there are some pits on the surface of silicon wafer caused by brittle removal of materials abrasive particles. The number of pits is large, but the size is small. There is only a few pits remain on the predicted wafer surface, because the effect of external ultrasonic vibration on the abrasive particles was only considered in the modeling process and most of the pits was removed by other abrasive particles. However, some additional vibrations that occur during the actual slicing process will cause the abrasive particles to impact the workpiece and form some crushing pits on the surface of the formed wafer surface, as shown in Fig.21.

The features in measured wafer surface morphology as shown in Fig. 21 is consistent with the features in Fig.22.By extracting the 1000 elements in a row of the workpiece topological matrix, the wafer surface contour can be obtained. Then the theoretical surface roughness values were calculated based on the surface contour. By extracting the elements of different rows for multiple calculations, the average value was taken as the final theoretical value of surface roughness value.

As shown in Table 2, the experimental values and simulation values are averaged by multiple measurements. The maximum error between theoretical surface roughness value and the experimental surface roughness value is $11.9 \%$ and the average error is $7.6 \%$, which means that the predicted results are close to the experimental results. The feasibility and correctness of the prediction method was verified by the results above. Moreover, the sampling contours determined by different removal modes can be mapped to the wafer surface model by using the dynamic contour sampling method, which achieves good prediction effect. 
Table 2 Surface roughness values $R a$

\begin{tabular}{cccc}
\hline Tests & Experimental mean value $(\mu \mathrm{m})$ & Theoretical mean value $(\mu \mathrm{m})$ & Error \\
\hline 1 & 0.75 & 0.78 & $4.0 \%$ \\
2 & 0.51 & 0.56 & $9.8 \%$ \\
3 & 0.46 & 0.50 & $8.7 \%$ \\
4 & 0.44 & 0.41 & $6.8 \%$ \\
5 & 0.42 & 0.37 & $11.9 \%$ \\
6 & 0.86 & 0.82 & $4.7 \%$ \\
7 & 1.34 & 1.47 & $9.7 \%$ \\
8 & 0.64 & 0.62 & $3.1 \%$ \\
9 & 0.43 & 0.46 & $7.0 \%$ \\
10 & 0.41 & 0.45 & $9.8 \%$ \\
\hline
\end{tabular}

\section{Discussion}

In this paper, a prediction model of wafer surface morphology has been established, and the simulation program of UAWS slicing single crystal silicon has been coded and validated by the experimental results. A series of simulation and experiments with different parameters were conducted for the study of wafer surface quality. In the following, the effects of wire saw speed $v_{s}$, feed rate $v_{w}$ and workpiece rotate speed $n_{w}$ will be presented.

Figs.23-25 illustrate the surface roughness value of wafer surface with different wire saw speeds, feed rates and workpiece rotate speed. It shows that both the experimental surface roughness value and the theoretical value decrease as the wire saw speed or the workpiece rotate speed increases, while the surface roughness value increases as the feed rate increases. This is because that there are more abrasive particles involved in the machining process in the same period with higher wire saw speed and higher workpiece rotate speed, which enhanced the cutting action of abrasive particles on the wafer surface. Nevertheless, higher feed rate will increase the cutting depth of the abrasive particles, thus increasing the surface roughness value. It can be seen that the theoretical results are in good agreement with the experimental results and the average error is $11.9 \%$.

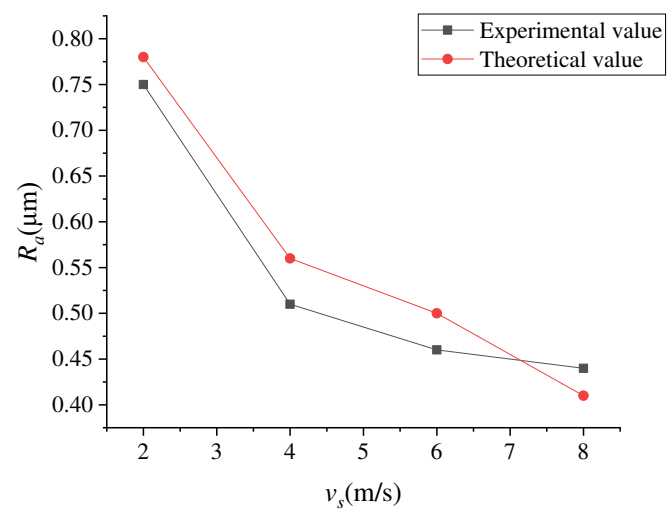

Fig.23 The surface roughness value with different wire saw speeds 


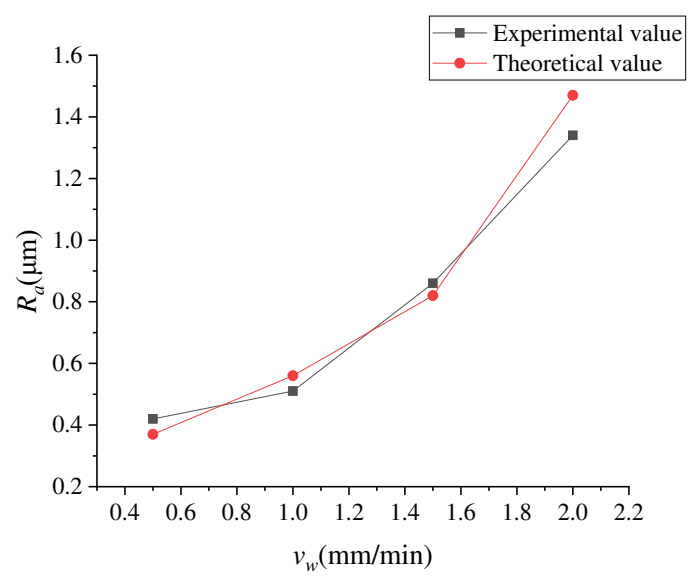

Fig.24 The surface roughness value with different feed rates

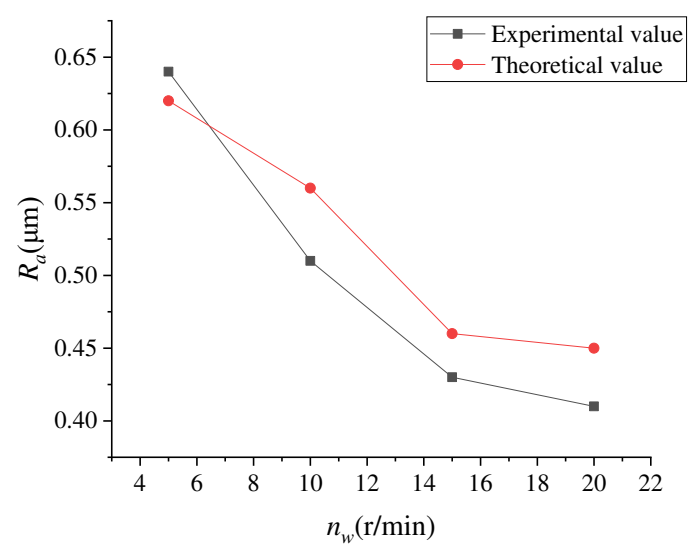

Fig.25 The surface roughness value with different workpiece rotate speeds

\section{Conclusions}

In this paper, a new model for prediction of wafer surface morphology in UAWS slicing single crystal silicon based on mixed material removal mode is presented. The validity of the prediction model has been confirmed by experiments.

1. The geometrical shape of the abrasive particle is simplified into a cone shape; the size distribution of abrasive particles conforms to normal distribution and the positions of abrasive particles are random. The surface model of diamond wire saw tool is established.

2. The trajectory equations of abrasive particles on the wire saw in different coordinate systems are set up from the perspective of kinematics. Then the mixed material removal model in wire saw slicing single crystal silicon is established. Based on that, the prediction model of wafer surface morphology is established. This model can be used to predict the wafer surface morphology in UAWS slicing.

3. The predicted results were verified by experiments. It was found that the predicted wafer surface roughness value was close to the experimental surface roughness value, with an average error of $11.9 \%$. The feasibility and accuracy of the prediction model are verified. In addition, the 
effect of slicing parameters including wire saw speed, feed rate and workpiece rotation speed on the surface quality of sliced wafers are investigated. The results show that increasing the wire saw speed and workpiece rotation speed can improve the surface quality of the wafer. The prediction model can be used to optimize the selection of slicing parameters and further study the generative mechanism of wafer surface morphology in wire saw slicing.

\section{Acknowledgement}

The authors thank the Natural Science Foundation of Shanghai for supporting for the study.

\section{Declarations}

Funding: No funding was received for conducting this study.

Authors Contributions: Yan Wang: Conceptualization, Methodology, Supervision, WritingReview \& Editing. Rui Wang: Formal analysis, Visualization, Writing-Original Draft. Shusheng Li: Resources, Data curation. Jianguo Liu: Resources, Investigation. Lixing Song: Investigation, Data curation

Data availability: The manuscript has no associated data or the data will not be deposited.

\section{Compliance with ethical standards}

Conflict of interest: The authors declare that they have no competing interests.

Ethical approval: This work has not been published elsewhere.

Consent to Participate: Not applicable.

Consent to publish: Not applicable.

\section{References}

[1] Yu XG, Wang P, Li XQ, Yang DR (2012) Thin Czochralski silicon solar cells based on diamond wire sawing technology. Sol Energy Mater Sol Cells. 98:337-342. https://doi.org/10.1016/j.solmat.2011.11.028

[2] Bhagavat S, Kao I (2006) Theoretical analysis on the effects of crystal anisotropy on wire sawing process and application to wafer slicing. Int J Mach Tools Manuf. 46(5):531-541. https://doi.org/10.1016/j.ijmachtools.2005.07.003

[3] Wu H (2016) Wire sawing technology: A state-of-the-art review. Precis Eng. (43):1-9. https://doi.org/10.1016/j.precisioneng.2015.08.008

[4] Li L, Li SJ, Tang AF, Li Y (2016) Influence of diamond wire saw excited by transverse ultrasonic vibration on cutting force and critical cutting depth of hard and brittle materials. J Mech Eng. 52(3):187-196. https://doi.org/10.3901/JME.2016.03.187

[5] Chung CH, Nhat LV (2014) Generation of diamond wire sliced wafer surface based on the distribution of diamond grits. Int J Precis Eng Man. 15(5):789-796. https://doi.org/10.1007/s12541-014-0401-2

[6] Ge MR, Bi WB, Ge PQ, Bi YC (2016) Experimental research on KDP crystal slicing with resin bonded diamond abrasive wire saw. Int J Adv Manuf Technol. 87(5-8):1671-1676. https://doi.org/ 10.1007/s00170-016-8577-5 
[7] Teomete E (2011) Effect of process parameters on surface quality for wire saw cutting of alumina ceramic. Gazi University Journal of Science. 24(2):297-297.

[8] Li SJ, Wan B, Landers RG (2013) Surface roughness optimization in processing $\mathrm{SiC}$ monocrystal wafers by wire saw machining with ultrasonic vibration. P I Mech Eng B-J Eng. 228(5):725-739. https://doi.org/10.1177/0954405413508116

[9] Gao YF, Ge PQ, Liu TY (2016) Experiment study on electroplated diamond wire saw slicing single crystal silicon. Mater Sci Semicond Process. (56):106-114.

https://doi.org/10.1016/j.mssp.2016.08.003

[10] Li XY, Gao YF, Ge PQ, Zhang L, Bi WB (2019) The effect of cut depth and distribution for abrasives on wafer surface morphology in diamond wire sawing of PV polycrystalline silicon. Mater Sci Semicond Process. (91):316-326. https://doi.org/10.1016/j.mssp.2018.12.004

[11] Wang J, Liu JB, Xiao YH, Zhao SC (2014) Simulation research on cutting brittle optical material with diamond wire saw based on LS-DYNA. Proceedings of SPIE. https://doi.org/10.1117/12.2069794

[12] Costa EC, Xavier FA, Knoblauch R, Binder C, Weingaertner WL (2020) Effect of cutting parameters on surface integrity of monocrystalline silicon sawn with an endless diamond wire saw. Sol Energy. 207: 640-650 https://doi.org/10.1016/j.solener.2020.07.018

[13] Yin YK, Gao YF, Wang LY, Zhang L, Pu TZ (2021) Analysis of crack-free surface generation of photovoltaic polysilicon wafer cut by diamond wire saw. Sol Energy. 216: 245-258. https://doi.org/10.1016/J.SOLENER.2021.01.009

[14] Li S, Liu Y, Hou X, Gao X (2015) Analysis and modeling cutting force for sic monocrystal wafer processing. J Mech Eng. 51(23):189-204 https://doi.org/10.3901/JME.2015.23.189

[15] Bidiville A, Wasmer1 K, Michler1 J, Nasch PM, Meer MVD, Ballif C (2010) Mechanisms of wafer sawing and impact on wafer properties. Prog Photovolt. 18(8):563-572. https://doi.org/10.1016/j.solener.2020.07.018

[16] Lambropoulos JC, Jacobs SD, Ruckman J (1999) Material removal mechanisms from grinding to polishing. Ceramic Transaction. (102):113-128.

[17] Marshall DB, Lawn BR, Evans AG (1982) Elastic/Plastic indentation damage in ceramics: the lateral crack system. J Am Ceram Soc. 65(11):561-566. https://doi.org/10.1111/j.11512916.1982.tb10782.x

[18] Gao YF, Ge PQ, Zhang L, Bi WB (2019) Material removal and surface generation mechanisms in diamond wire sawing of silicon crystal. Mater Sci Semicond Process. (103):104642. https://doi.org/10.1016/j.mssp.2019.104642

[19] Ge MR, Zhu HT, Huang CZ, Liu A, Bi WB (2018) Investigation on critical crack-free cutting depth for single crystal silicon slicing with fixed abrasive wire saw based on the scratching machining experiments. Mater Sci Semicond Process. 74: 261-266. https://doi.org/10.1016/j.mssp.2017.10.027

[20] Xiao HP, Wang HR, Yu N, Liang RG, Tong Z, Chen Z, Wang JH (2019) Evaluation of fixed abrasive diamond wire sawing induced subsurface damage of solar silicon wafers. J Mater Process Technol. 273. https://doi.org/10.1016/j.jmatprotec.2019.116267

[21] Wang Y, Li DL, Liu JG, Peng SQ, Wang R (2018) Prediction and experimental verification of workpiece surface topology in axial ultrasonic vibration assisted grinding based on dynamic profile sampling method. J Mech Eng. 54(21):221-230. 
https://doi.org/10.3901/JME.2018.21.221

[22] Nguyen TA, Butler DL (2005) Simulation of precision grinding process, part 1: generation of the grinding wheel surface. Int J Mach Tools Manuf. 45(11):1321-1328.

https://doi.org/10.1016/j.ijmachtools.2005.01.005

[23] Nguyen TA, Butler DL (2005) Simulation of precision grinding process, part 2: the interaction of the abrasive grain with workpiece. Int J Mach Tools Manuf. (45):1329-1336.

https://doi.org/10.1016/j.ijmachtools.2005.01.006

[24] Ge MR, Zhu HT, Huang CZ, Liu A, Bi WB (2018) Investigation on critical crack-free cutting depth for single crystal silicon slicing with fixed abrasive wire saw based on the scratching machining experiments. Mater Sci Semicond Process. (74):261-266.

https://doi.org/10.1016/j.mssp.2017.10.027 